\title{
ON THE PASSIVITY OF A ONE-LINK RIGID MASTER - FLEXIBLE SLAVE MANIPULATOR
}

\author{
Takahiro Mori ${ }^{*}$ Yoshifumi Morita ${ }^{* *}$ Hiroyuki Ukai ${ }^{* *}$ \\ * Gifu National College of Technology, \\ Kamimakuwa, Motosu-city, Gifu 501-0495, JAPAN \\ ** Nagoya Institute of Technology, \\ Gokiso, Showa, Nagoya, 466-8555, JAPAN
}

\begin{abstract}
This paper discusses the passivity of a one degree-of-freedom rigid master - flexible slave (RMFS) manipulator. First of all, nonlinear motion equations of the RMFS system is derived as a distributed parameter system by using the Hamilton's principle. Applied the symmetric bilateral control to the RMFS system, the passivity of the system in the positioning operation is proved by using a Lyapunov function related to the total energy. Moreover, the steady states in pushing operation to an environment is discussed, then the passivity of the system in the neighborhood of the desired state is proved. Copyright@ 2005 IFAC
\end{abstract}

Keywords: master-slave system, flexible arm, passivity, symmetric bilateral control, distributed parameter system, Laypunov method

\section{INTRODUCTION}

From the early years of robotics, several control schemes of master-slave manipulators have been investigated. In particular, the master-slave manipulators have recently received a great deal of attention as one of telerobotics technologies. One of utilities of the master-slave manipulators is operation in the extreme environment such as nuclear power plants, outer space, disaster sites and so on( Special issues of Theory of Telerobotics, 1993). In these cases slave manipulators may consist of long and light-weight arms to secure the large working space. Since these manipulators have elastic deformations, they are regarded as flexible arms. On the other hand, a master arm is simple and rigid for the sake of human maneuverability. Such a master-slave system is called a rigid master - flexible slave (RMFS) system.
In general, it is difficult to strictly discuss the stability of a master-slave system, because both an operator and an environment are included in a system. From this reason it is significant to discuss the passivity of an input-output relation of a master-slave system(Yokokohji, 1993). Yokokohji and Yoshikawa represented the input-output relation of a master-slave system as the two-terminalport network model and proposed a controller based on the passivity(Yokokohji and Yoshikawa, 1994). Moreover, from the viewpoint of passivity, Kosuge et al. proposed an alternative control algorithm for a scaled tele-manipulation system based on a task-oriented virtual tool(Kosuge et al., 1995). The stability of the resultant system was analyzed on the basis of the passivity of the system and the total stability was guaranteed for a human operator and a passive environment. 
However, in case of a flexible master-slave system in contrast to the case of a rigid master-slave system, elastic vibration modes of flexible arms may cause the instability of a total system. Moreover, the mathematical model of flexible systems is very complicated. These reasons make the passivity issue in case of flexible systems difficult.

On the other hand, the the passivity based control method of flexible manipulators has been recently discussed(Morita et al., 2002; Matsuno and Endo, 2004). Osuka and Matsuno discussed on robust passivity of multi-link-flexible manipulators(Osuka and Matsuno, 1993). It was shown that when the joint angular velocity and the joint torque are regarded as the input variable and the output variable, respectively, the control system from the input to the output becomes robust stable by using control law based on passivity. However the passivity of master-slave manipulators with flexible slave arms have not been investigated.

In this paper we study on the passivity of one degree-of-freedom RMFS system under the symmetric bilateral control configuration. A distributed parameter model of RMFS system is derived by using Hamilton's principle, which consist of a couple of ordinary differential equations of master and slave angles and a partial differential equation with the boundary conditions of the bending vibration of a slave arm. The passivity of the RMFS system is proven in cases of both the positioning and the pushing operation by using Lyapunov method.

\section{DISTRIBUTED PARAMETER MODEL}

The one o.d.f master-slave system treated in this paper is shown in Fig.1 and Fig.2. Each arm rotates on the horizontal plain. The slave system has a long arm, hence it causes elastic deformation. A human operates the slave arm by handling the master arm while watching the movement of the slave arm. It is assumed that the communication time lag does not exist in this system.

Let $O_{m}-X_{m} Y_{m}$ denotes an inertial Cartesian coordinate frame of a master arm. $J_{m}$ denotes the moment of inertia of the rotor of the motor and the master arm. $\theta_{m}(t)$ and $\tau_{m}(t)$ are the angle of rotation of the master motor and the torque developed by the master motor, respectively. $\tau_{h}(t)$ denotes the torque applied by the human operator.

Let $O_{s}-X_{s} Y_{s}$ denote an inertial Cartesian coordinate frame of a slave arm. The motor actuating

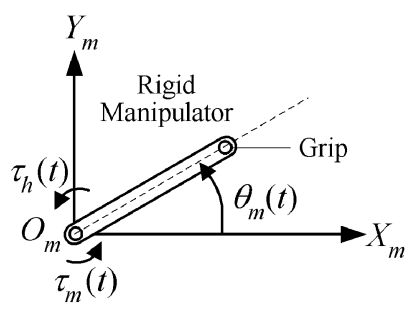

Fig. 1. Master Manipulator

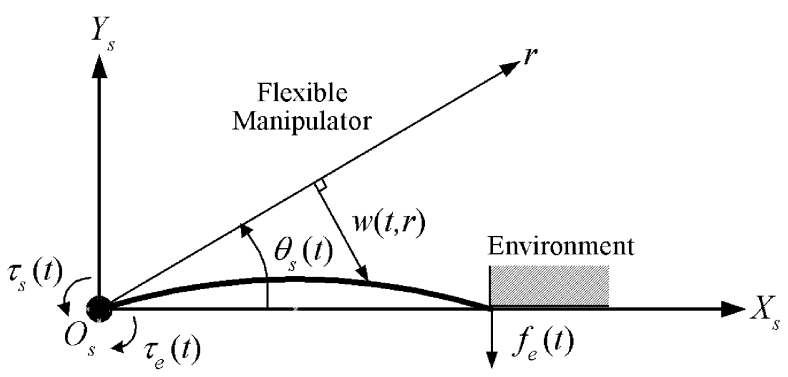

Fig. 2. Slave Manipulator

a slave arm has the moment of inertia $J_{s}$ around the shaft of the rotor. The slave arm of length $L$, having uniform mass density $\rho$ per unit length and uniform bending rigidity $E I$, is fixed at the rotor of the motor. Let $\theta_{s}(t)$ and $\tau_{s}(t)$ be the angles of rotation of the slave arm and the torque developed by the slave motor, respectively. $w(t, r)$ denotes the bending displacement at time $t$ and at a spatial point $r(0 \leq r \leq L)$. $f_{e}(t)$ denotes the reaction force received from an environment at the tip of the slave arm, and $\tau_{e}(t)$ denotes the reaction torque corresponding to the force $f_{e}(t), \tau_{e}(t)=L f_{e}(t)$. The positive direction of the variables are indicated with arrows in Fig.1 and Fig.2.

The total kinetic energy $\mathcal{T}$ and the potential energy $\mathcal{U}$ are given by

$$
\begin{aligned}
\mathcal{T} & =\mathcal{T}_{m}+\mathcal{T}_{s-\text { motor }}+\mathcal{T}_{s-\text { arm }}, \\
\mathcal{T}_{m} & =\frac{1}{2} J_{m} \dot{\theta}_{m}^{2}(t), \\
\mathcal{T}_{s-\text { motor }} & =\frac{1}{2} J_{s} \dot{\theta}_{s}^{2}(t), \\
\mathcal{T}_{s-a r m} & =\frac{1}{2} \int_{0}^{L} \rho\left(r \dot{\theta}_{s}(t)-\dot{w}(t, r)\right)^{2} d r, \\
\mathcal{U} & =\frac{1}{2} \int_{0}^{L} E I\left(w^{\prime \prime}(t, r)\right)^{2} d r,
\end{aligned}
$$

where $w_{L}(t)=w(t, L), w_{L}^{\prime}(t)=w^{\prime}(t, L), \mathcal{T}_{m}$ is the rotational kinetic energy of the master arm, $\mathcal{T}_{s-\text { motor }}$ is the rotational kinetic energy of the slave motor, $\mathcal{T}_{s-a r m}$ is the translational kinetic energy of the slave arm, and $\mathcal{U}$ is the bending 
strain energy of the slave arm. $\left({ }^{\prime}\right)$ and $\left({ }^{\prime}\right)$ denote the time derivative and the derivative with respect to the spatial variable $r$, respectively. The virtual work $\delta \mathcal{W}$ by external torques is given by

$$
\begin{aligned}
\delta \mathcal{W} & =\tau_{m}(t) \delta \theta_{m}(t)+\tau_{s}(t) \delta \theta_{s}(t) \\
& +\tau_{h}(t) \delta \theta_{m}(t)+\tau_{e}(t)\left\{-\left(\delta \theta_{s}-(1 / L) \delta w_{L}(t)\right)\right\} .
\end{aligned}
$$

By applying Hamilton's principle, the equations of motion are derived as follows:

Equation of the master arm:

$$
J_{m} \ddot{\theta}_{m}(t)=\tau_{m}(t)+\tau_{h}(t),
$$

Equation of the rotation of the slave arm:

$$
\begin{aligned}
J_{s} \ddot{\theta}_{s}+E I w_{0}^{\prime \prime}= & \tau_{s}-\rho \int_{0}^{L}\left(2 w_{r} \dot{w}_{r} \dot{\theta}_{s}+w_{r}^{2} \ddot{\theta}_{s}\right) d r \\
& +\rho \dot{\theta}_{s}^{2} \int_{0}^{L} r w_{r} d r
\end{aligned}
$$

Equation of the bending vibration of the slave arm:

$$
\begin{aligned}
& \ddot{w}(t, r)+\frac{E I}{\rho} w^{\prime \prime \prime \prime}(t, r)-r \ddot{\theta}_{s}(t)-\dot{\theta}_{s}^{2} w_{r}=0,(9) \\
& E I w_{L}^{\prime \prime \prime}(t)=-\tau_{e}(t) / L, \\
& w_{L}^{\prime \prime}(t)=w(t, 0)=w^{\prime}(t, 0)=0 .
\end{aligned}
$$

\section{PASSIVITY OF THE RMFS SYSTEM}

The passivity is deeply related to a input-output relation of a system. Moreover, in the masterslave system, the control configuration should be pre-determined because both arms are connected through the control system. In general, the input is an operator input torque. Therefore key points proving the passivity are how to determine a output and a control configuration. In the following sections, we prove the passivity for both the positioning operation and pushing operation under a certain control configuration.

\subsection{Passivity in positioning operation}

The control purpose of the positioning operation is to operate a master arm so that the angle of the slave arm $\theta_{s}(t)$ reaches the desired angle $\theta_{d}$ in the steady state. Therefore, in this case, we discuss the passivity of the RMFS system under the condition $\tau_{e}(t)=0$.
[Theorem 1] Assume that the symmetric bilateral control is used, whose control torques are represented as

$$
\begin{aligned}
\tau_{m}(t) & =k_{m}\left(\theta_{s}(t)-\theta_{m}(t)\right), \\
\tau_{s}(t) & =k_{s}\left(\theta_{m}(t)-\theta_{s}(t)\right),
\end{aligned}
$$

where $k_{m}$ and $k_{s}$ are proportional gains.

Then the following input-output relation of the RMFS system is passive.

$$
\int_{0}^{t} \dot{\theta}_{m}(\tau) \tau_{h}(\tau) d \tau \geq-\gamma^{2}
$$

where $\gamma$ is a positive constant.

\section{(Proof)}

Define the following modified energy function.

$$
\begin{aligned}
V(\boldsymbol{x}(t, r))= & k_{1} \mathcal{T}_{m}+k_{2}\left(\mathcal{T}_{s-\text { motor }}+\mathcal{T}_{s-a r m}+\mathcal{U}\right) \\
& +\frac{1}{2} k_{3}\left(\theta_{m}(t)-\theta_{s}(t)\right)^{2}
\end{aligned}
$$

where $k_{1}, k_{2}$, and $k_{3}$ are positive constants, and $\boldsymbol{x}(t, r)=\left[\theta_{m}(t), \dot{\theta}_{m}(t), \theta_{s}(t), \dot{\theta}_{s}(t), w(t, r)\right.$, $\dot{w}(t, r)]^{T}$. The first term of the right hand side in (15) implies the energy of the master manipulator weighted by $k_{1}$, and the second term the total energy of the slave manipulator weighted by $k_{2}$. The last term is added as a pseudo-energy to insure that steady state condition. Since the conditions of the steady states are expressed as $\dot{\theta}_{m}(t)=0$, $\dot{\theta}_{s}(t)=0, \dot{w}(t, r)=0$, the desired state $\boldsymbol{x}_{d}=\left[\theta_{d}, 0\right.$, $\left.\theta_{d}, 0,0,0\right]^{T}$ is a unique minimum of $V$. It is straightforward to check that the positiveness of the constants $k_{1}, k_{2}$, and $k_{3}$ in (15) guarantees $V(\boldsymbol{x}(t, r)) \geq 0$ and that the global minimum of $V(\boldsymbol{x}(t, r))=0$ is attained only at the desired state $\boldsymbol{x}_{d}$. This implies that $V(\boldsymbol{x}(t, r))$ is a Lyapunov function candidate.

By differentiating (15) with respect to $t$ along the equations of motion $(8) \sim(11)$, we obtain ; the first term:

$$
\dot{\mathcal{T}}_{m}=J_{m} \dot{\theta}_{m} \ddot{\theta}_{m}=\left(J_{m} \ddot{\theta}_{m}\right) \dot{\theta}_{m}=\left(\tau_{m}+\tau_{h}\right) \dot{\theta}_{m}(16)
$$

the second term:

$$
\begin{aligned}
& \dot{\mathcal{T}}_{s-\text { motor }}+\dot{\mathcal{T}}_{s-a r m}+\dot{\mathcal{U}} \\
& =\dot{\theta}_{s}\left\{\tau_{s}-E I w_{0}^{\prime \prime}-\rho \int_{0}^{L}\left(2 w_{r} \dot{w}_{r} \dot{\theta}_{s}+w_{r}^{2} \ddot{\theta}_{s}\right) d r\right. \\
& \left.+\rho \dot{\theta}_{s}^{2} \int_{0}^{L} r w_{r} d r\right\}+\dot{\theta}_{s}\left\{\rho \int_{0}^{L}\left(2 w_{r} \dot{w}_{r} \dot{\theta}_{s}+w_{r}^{2} \ddot{\theta}_{s}\right) d r\right.
\end{aligned}
$$




$$
\begin{aligned}
& \left.+E I w_{0}^{\prime \prime}-\rho \dot{\theta}_{s}^{2} \int_{0}^{L} r w_{r} d r\right\}-E I \int_{0}^{L} \dot{w}_{r}^{\prime \prime \prime \prime} w_{r} d r \\
& +E I \int_{0}^{L} \dot{w}_{r}^{\prime \prime \prime \prime} w_{r} d r=\dot{\theta}_{s} \tau_{s},
\end{aligned}
$$

and the last term:

$$
\begin{aligned}
\frac{d}{d t}\left\{\frac{1}{2}\left(\theta_{m}-\theta_{s}\right)^{2}\right\} & =\left(\theta_{m}-\theta_{s}\right)\left(\dot{\theta}_{m}-\dot{\theta}_{s}\right) \\
& =\dot{\theta}_{m}\left(\theta_{m}-\theta_{s}\right)-\dot{\theta}_{s}\left(\theta_{m}-\theta_{s}\right) .
\end{aligned}
$$

As the result, we have the following equation.

$$
\begin{aligned}
\dot{V}(\boldsymbol{x}(t, r))= & k_{1} \dot{\mathcal{T}}_{m}+k_{2}\left(\dot{\mathcal{T}}_{s-m o t o r}+\dot{\mathcal{T}}_{s-a r m}+\dot{\mathcal{U}}\right) \\
& +k_{3} \frac{d}{d t}\left\{\frac{1}{2}\left(\theta_{m}-\theta_{s}\right)^{2}\right\} \\
= & k_{1}\left(\tau_{m}+\tau_{h}\right) \dot{\theta}_{m}+k_{2} \dot{\theta}_{s} \tau_{s} \\
& +k_{3} \dot{\theta}_{m}\left(\theta_{m}-\theta_{s}\right)-k_{3} \dot{\theta}_{s}\left(\theta_{m}-\theta_{s}\right) \\
= & \dot{\theta}_{m}\left\{k_{1}\left(\tau_{m}+\tau_{h}\right)+k_{3}\left(\theta_{m}-\theta_{s}\right)\right\} \\
& +\dot{\theta}_{s}\left\{k_{2} \tau_{s}-k_{3}\left(\theta_{m}-\theta_{s}\right)\right\}
\end{aligned}
$$

By substituting (12) and (13) into (19) and setting $k_{m}=k_{3} / k_{1}$ and $k_{s}=k_{3} / k_{2}$, we have

$$
\frac{d V(\boldsymbol{x}(t, r))}{d t}=k_{1} \dot{\theta}_{m}(t) \tau_{h}(t) .
$$

Equation (20) yields the product of the human operator torque $\tau_{h}(t)$ and the angular velocity of the master arm $\theta_{m}(t)$. Therefore, it is concluded that the passivity between the input and the output is proven as follows:

$$
\begin{aligned}
\int_{0}^{t} \frac{d V(\boldsymbol{x}(\tau, r))}{d t} d \tau & =V(\boldsymbol{x}(t, r))-V(\boldsymbol{x}(0, r)) \\
& \geq-V(\boldsymbol{x}(0, r)) \geq-\gamma^{2}, \quad
\end{aligned}
$$

(Q.E.D.)

\subsection{Passivity in pushing operation}

We consider the passivity in the case of the pushing operation using the RMFS system with the symmetric bilateral control. The control purpose in this case is to operate a master arm so as to push the environment with a desired torque $\tau_{e}^{d}$ using the slave arm.

First of all, we consider steady states of the RMFS system when a tip of slave arm pushes an environment with a desired torque.

\section{[Lemma 1]}

Applied the symmetric bilateral control, the RMFS system in pushing operation with a desired torque $\tau_{e}^{d}$ has the steady state as follows:

$$
\theta_{m}(t)=\theta_{m}^{d}, \theta_{s}(t)=\theta_{s}^{d}, w(t, r)=w^{d}(r),
$$

where,

$$
\begin{gathered}
\theta_{m}^{d}=\left(\frac{1}{k_{s}}+\frac{L}{3 E I}\right) \tau_{e}^{d}, \quad \theta_{s}^{d}=\frac{L}{3 E I} \tau_{e}^{d} \\
w^{d}(r)=\frac{\tau_{e}^{d}}{6 E I L}\left(3 L r^{2}-r^{3}\right),
\end{gathered}
$$

Moreover, the steady operator input torque $\tau_{h}^{d}$ is given by

$$
\tau_{h}^{d}=\frac{k_{m}}{k_{s}} \tau_{e}^{d}
$$

\section{(Proof)}

The conditions that the RMFS system is the steady state are $\dot{\theta}_{m}(t)=0, \dot{\theta}_{s}(t)=0, \dot{w}(t, r)=0$. It is assumed that the reaction torque of the slave arm $\tau_{e}$ is given by the desired torque $\tau_{e}^{d}$. By integrating (9) with boundary conditions (10) and (11), we have

$$
w^{\prime \prime}(r)=c_{1} r+c_{2} \equiv w^{\prime \prime}(r),
$$

where

$$
c_{1}=-\frac{\tau_{e}^{d}}{E I L}, \quad c_{2}=\frac{\tau_{e}^{d}}{E I} .
$$

By integrating again the above equation with the boundary condition (11), we obtain

$$
w(r) \equiv w^{d}(r)=\frac{1}{6} c_{1} r^{3}+\frac{1}{2} c_{2} r^{2} .
$$

From the above equations,

$$
w^{d}(L)=\frac{L^{2}}{3 E I} \tau_{e}^{d}, \quad w^{\prime \prime}{ }^{d}(0)=\frac{\tau_{e}^{d}}{E I} .
$$

Since the relation $\theta_{s}=w(L) / L$ holds,

$$
\theta_{s}^{d}=\frac{w^{d}(L)}{L}=\frac{L}{3 E I} \tau_{e}^{d}
$$

By substituting $\tau_{s}=k_{s}\left(\theta_{m}-\theta_{s}\right)$ to the steady state relation of (8), and using the relations (26) and (27), we conclude

$$
\theta_{m}^{d}=\frac{\tau_{e}^{d}}{k_{s}}+\theta_{s}^{d}=\left(\frac{1}{k_{s}}+\frac{L}{3 E I} \tau_{e}^{d}\right)
$$

Moreover since $\tau_{m}=k_{m}\left(\theta_{s}-\theta_{m}\right)$ and $\tau_{h}=-\tau_{m}$ in the steady state, the above equation yields

$$
\tau_{h}^{d}=\frac{k_{m}}{k_{s}} \tau_{e}^{d}
$$


This lemma explains that the symmetric bilateral control enables an operator to perform the pushing operation while receiving steadily the torque at the tip of the slave arm from the environment.

Now we consider the passivity of the RMFS system for small deviations from the steady states. By linearlizing (7) (11) around the desired states, the linear equations in the neighborhood of the desired state are obtained as follows:

$$
\begin{aligned}
& J_{m} \delta \ddot{\theta}_{m}(t)=\delta \tau_{m}(t)+\delta \tau_{h}(t), \\
& J_{s} \delta \ddot{\theta}_{s}(t)+E I \delta w^{\prime \prime}(t, 0)=\delta \tau_{s}(t), \\
& \delta \ddot{w}(t, r)+\frac{E I}{\rho} \delta w^{\prime \prime \prime \prime}(t, r)=r \delta \ddot{\theta}_{s}(t), \\
& E I \delta w^{\prime \prime \prime}(t, L)=-\delta \tau_{e} / L, \\
& \delta w^{\prime \prime}(t, L)=\delta w(t, 0)=\delta w^{\prime}(t, 0)=0 .
\end{aligned}
$$

Then we obtain the following result.

\section{[Theorem 2]}

The following input-output relation of the RMFS system in the pushing operation is passive under the symmetric bilateral control.

$$
\int_{0}^{t}\left[k_{1} \delta \dot{\theta}_{m}(t),-k_{2} \delta \dot{\theta}_{s}(t)\right]\left[\begin{array}{l}
\delta \tau_{h}(t) \\
\delta \tau_{e}(t)
\end{array}\right] d t \geq-\gamma^{2},
$$

where $\gamma$ is positive constant.

\section{(Proof)}

Define the following Lyapunov function candidate:

$$
\begin{aligned}
V(\delta \boldsymbol{x}(t, r))= & k_{1} \delta \mathcal{T}_{m}+k_{2}\left(\delta \mathcal{T}_{s-\text { motor }}+\delta \mathcal{T}_{s-a r m}+\delta \mathcal{U}\right) \\
& +\frac{1}{2} k_{3}\left(\delta \theta_{m}(t)-\delta \theta_{s}(t)\right)^{2},
\end{aligned}
$$

where $k_{1}, k_{2}$, and $k_{3}$ are positive constants, and $\delta \mathcal{T}_{m}, \delta \mathcal{T}_{s-m o t o r}, \delta \mathcal{T}_{s-a r m}$, and $\delta \mathcal{U}$ are the deviations of the energies from the equilibrium states, which are expressed as follows:

$$
\begin{aligned}
\delta \mathcal{T}_{m} & =\frac{1}{2} J_{m} \delta \dot{\theta}_{m}^{2}(t) \\
\delta \mathcal{T}_{s-\text { motor }} & =\frac{1}{2} J_{s} \delta \dot{\theta}_{s}^{2}(t) \\
\delta \mathcal{T}_{s-\text { arm }} & =\frac{1}{2} \int_{0}^{L} \rho\left(r \delta \dot{\theta}_{s}(t)-\delta \dot{w}(t, r)\right)^{2} d r,( \\
\delta \mathcal{U} & =\frac{1}{2} \int_{0}^{L} E I\left(\delta w^{\prime \prime}(t, r)\right)^{2} d r
\end{aligned}
$$

The deviation of state variable is expressed as $\delta \boldsymbol{x}(t, r)=\left[\delta \theta_{m}(t), \delta \dot{\theta}_{m}(t), \delta \theta_{s}(t), \delta \dot{\theta}_{s}(t), \delta w(t, r)\right.$, $\delta \dot{w}(t, r)]^{T}$. It is straightforward to check that the positiveness of the constants $k_{1}, k_{2}$, and $k_{3}$ in (36) guarantees $V(\delta \boldsymbol{x}) \geq 0$ and that the global minimum of $V(\delta \boldsymbol{x})=0$ is attained only at the desired states $\delta \boldsymbol{x}_{d}=0$.

In the same manner as Theorem 1, by differentiating (36) with respect to $t$, employing the linearized equations of motion (30) (34), and using the approximating relation that $w(t, L) \ll L$, we obtain

$$
\begin{aligned}
& \frac{d V(\delta \boldsymbol{x}(t, r))}{d t}=\delta \dot{\theta}_{m}(t)\left\{k_{1}\left(\delta \tau_{m}(t)+\delta \tau_{h}(t)\right)\right. \\
& \left.+k_{3}\left(\delta \theta_{m}(t)-\delta \theta_{s}(t)\right)\right\}+\delta \dot{\theta}_{s}(t)\left\{k_{2}\left(\delta \tau_{s}(t)-\delta \tau_{e}(t)\right)\right. \\
& -k_{3}\left(\delta \theta_{m}(t)-\delta \theta_{s}(t)\right\} .
\end{aligned}
$$

By substituting (12) and (13) into (41) and setting $k_{m}=k_{3} / k_{1}$ and $k_{s}=k_{3} / k_{2}$, we finally obtain

$$
\frac{d V(\delta \boldsymbol{x}(t, r))}{d t}=\left[k_{1} \delta \dot{\theta}_{m}(t),-k_{2} \delta \dot{\theta}_{s}(t)\right]\left[\begin{array}{l}
\delta \tau_{h}(t) \\
\delta \tau_{e}(t)
\end{array}\right](42)
$$

where the minus sign of $\delta \dot{\theta}_{s}$ is due to setting the direction of the slave angular velocity $\dot{\theta}_{s}(t)$ opposite against to the reaction torque $\tau_{e}(t)$. Equation (42) yields the product of the vectors $\left[\delta \tau_{h}(t) \delta \tau_{e}(t)\right]^{T}$ and $\left[k_{1} \delta \dot{\theta}_{m}(t)-k_{2} \delta \dot{\theta}_{s}(t)\right]^{T}$. Therefore, it is concluded that the passivity between the input and the output is proven as follows:

$$
\begin{aligned}
\int_{0}^{t} \frac{d V(\delta \boldsymbol{x}(t, r))}{d t} d t & =V(\delta \boldsymbol{x}(t, r))-V(\delta \boldsymbol{x}(0)) \\
& \geq-V(\delta \boldsymbol{x}(0)) \geq-\gamma^{2}
\end{aligned}
$$

The feedback control given by (12) and (13) is called the "symmetric bilateral control" (Anderson and Spong, 1989). It was shown that in a rigid master-slave system the "symmetric bilateral control" gives the passivity(Burnett, 1957; Yokokohji, 1993). These two theorems show the general extension of this well-known result; that is, even if the slave arm has flexibility, the a master- slave system with symmetric bilateral control is passive.

\section{NUMERICAL SIMULATIONS}

In order to verify the passivity of RMFS using the symmetric bilateral control some numerical simulation results are shown in case of the positioning operation. Fig.1 shows the transient responses of the angles of the master arm and the slave arm, and Fig.2 the transient response of the bending 
moment of slave arm. In this case, it is assumed that the anglar velosity of the master arm $\dot{\theta}_{m}$ is fedback to the operator torque $\tau_{h}$ under the assumption that a human operator is represented as a suitable impedance model. It is found that all state variables converge to desired states stablely. In the similar manner, we can show the stability of the whole system in case of the pussing operation under the suitable impedance model of an environment.

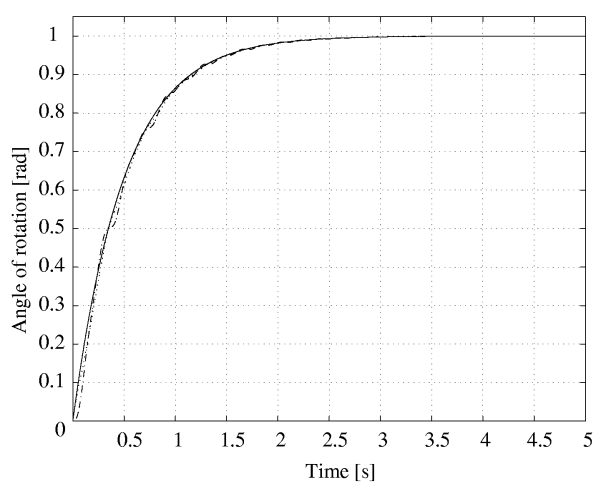

Fig. 3. Transient responses of the angles $\left(\theta_{m}^{d}\right.$ :solid line, $\theta_{m}(t)$ :dotted line, and $\theta_{s}(t)$ :dashed line)

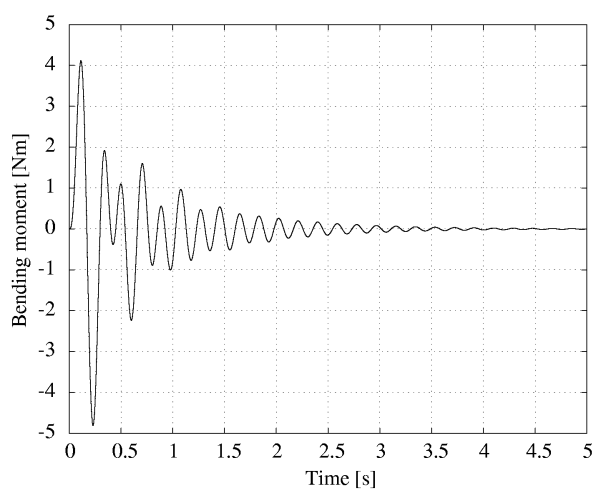

Fig. 4. Transient response of the bending moment

\section{CONCLUSION}

We investigated the passivity of the RMFS system in cases of both the positioning and the pushing operations.

As mentioned before, it is difficult to discuss the stability of a master-slave system. The situation in case of flexible system is same. However, the obtained results guarantee that the discussions for the stability of a master-slave system is similar to the case of rigid system. This gives benefit to further discussions for the control design based on the passivity. Of course, it is indispensable to consider the suppression control for elastic vibrations of slave arms. However, it is very important for the control design that the passivity result is based on the inherent kinetic structure of a considered system.

In the future work we apply these results to design methods of a new bilateral control based on the passivity considering the absorption of the elastic vibration.

\section{ACKNOWLEDGEMENT}

This work was partially supported by the Ministry of Education, Science, Sports and Culture, Grantin-Aid for Scientific Research (C), 15560218, 2003.

\section{REFERENCES}

Special Issues of Theory of Tele-robotics (1993), Journal of Robotics Society of Japan, 11(6) (in Japanese).

Y. Yokokohji (1993), Control Theory of Masterslave System, Journal of Robotics Society of Japan, 11(6), 26-34 (in Japanes).

R. J. Anderson and M. W. Spong (1989), Bilateral control of teleoperators with time delay, $E E E$ Trans. on Automatic Control, 34(5), 494501.

Y. Morita, F. Matsuno, Y. Kobayashi, M. Ikeda, H. Ukai, and H. Kando (2002), LyapunovBased Force Control of a Flexible Arm Considering Bending and Torsional Deformation, Proc. 15th IFAC World Congres, Session Slot: T-Tu-M04, No1.

F. Matsuno and T. Endo (2004), Dynamics based Control of Two-Link Flexible Arm, Proc, the IEEE International Workshop on Advanced Motion Control, 135-140.

K. Osuka and F. Matsuno (1999), On Robustness of Passivity of Manipulator, Journal of Robotics Society of Japan, 19(1), 75-80 (in Japanese).

Y. Yokokohji and T. Yoshikawa (1994), Bilateral Control of Master-Slave Manipulators of Ideal Kinesthetic Counpling-Formulation and Experiment, IEEE Trans. Robotics and Automation, 10(4), 605-620.

K. Kosuge, T. Itoh and T. Fukuda (1995), Taskoriented Control of Scaled Telemanipulation System, Trans. IEEJ, 115(10), 1121-1127 (in Japanese).

J.R.Burnett (1957), Force-Refrecting Servos Add "Feel" to Remote Controls, Control Engineering. 4(7), 1269-1274. 